\title{
Improving the estimates for a sequence involving prime numbers
}

\author{
Christian Axler \\ Department of Mathematics, University of Düsseldorf \\ 40225 Düsseldorf, Germany \\ e-mail: christian.axler@hhu.de
}

Received: 24 September 2018

Accepted: 18 December 2018

\begin{abstract}
Based on new explicit estimates for the prime counting function, we improve the currently known estimates for the particular sequence $C_{n}=n p_{n}-\sum_{k \leq n} p_{k}, n \geq 1$, involving the prime numbers.
\end{abstract}

Keywords: Prime counting function, Prime numbers, Sum of primes.

2010 Mathematics Subject Classification: 11N05, 11A41.

\section{Introduction}

Let $p_{n}$ denote the $n$th prime number. In this paper, we consider the sequence $\left(C_{n}\right)_{n \geq 1}$, where

$$
C_{n}=n p_{n}-\sum_{k \leq n} p_{k}
$$

In [1, Theorem 10], it is proved that the asymptotic formula

$$
C_{n}=\sum_{k=1}^{m-1}(k-1) !\left(1-\frac{1}{2^{k}}\right) \frac{p_{n}^{2}}{\log ^{k} p_{n}}+O\left(\frac{p_{n}^{2}}{\log ^{m} p_{n}}\right)
$$

holds for each positive integer $m$. By setting $m=9$ in (1.1), we get

$$
C_{n}=\frac{p_{n}^{2}}{2 \log p_{n}}+\frac{3 p_{n}^{2}}{4 \log ^{2} p_{n}}+\frac{7 p_{n}^{2}}{4 \log ^{3} p_{n}}+\chi(n)+O\left(\frac{p_{n}^{2}}{\log ^{9} p_{n}}\right)
$$


where

$$
\chi(n)=\frac{45 p_{n}^{2}}{8 \log ^{4} p_{n}}+\frac{93 p_{n}^{2}}{4 \log ^{5} p_{n}}+\frac{945 p_{n}^{2}}{8 \log ^{6} p_{n}}+\frac{5715 p_{n}^{2}}{8 \log ^{7} p_{n}}+\frac{80325 p_{n}^{2}}{16 \log ^{8} p_{n}} .
$$

In the direction of (1.2), the present author [1, Theorems 3 and 4] showed that

$$
C_{n} \geq \frac{p_{n}^{2}}{2 \log p_{n}}+\frac{3 p_{n}^{2}}{4 \log ^{2} p_{n}}+\frac{7 p_{n}^{2}}{4 \log ^{3} p_{n}}+\Theta(n)
$$

for every integer $n \geq 52703656$, where

$$
\Theta(n)=\frac{43.6 p_{n}^{2}}{8 \log ^{4} p_{n}}+\frac{90.9 p_{n}^{2}}{4 \log ^{5} p_{n}}+\frac{927.5 p_{n}^{2}}{8 \log ^{6} p_{n}}+\frac{5620.5 p_{n}^{2}}{8 \log ^{7} p_{n}}+\frac{79075.5 p_{n}^{2}}{16 \log ^{8} p_{n}},
$$

and that the upper bound

$$
C_{n} \leq \frac{p_{n}^{2}}{2 \log p_{n}}+\frac{3 p_{n}^{2}}{4 \log ^{2} p_{n}}+\frac{7 p_{n}^{2}}{4 \log ^{3} p_{n}}+\Omega(n)
$$

holds for every positive integer $n$, where

$$
\Omega(n)=\frac{46.4 p_{n}^{2}}{8 \log ^{4} p_{n}}+\frac{95 \cdot 1 p_{n}^{2}}{4 \log ^{5} p_{n}}+\frac{962.5 p_{n}^{2}}{8 \log ^{6} p_{n}}+\frac{5809.5 p_{n}^{2}}{8 \log ^{7} p_{n}}+\frac{118848 p_{n}^{2}}{16 \log ^{8} p_{n}} .
$$

Using new explicit estimates for the prime counting function $\pi(x)$, see [2, Propositions 3 and 5], we improve the inequalities (1.3) and (1.4) by showing the following two results.

Theorem 1.1. For every integer $n \geq 440200309$, we have

$$
C_{n} \geq \frac{p_{n}^{2}}{2 \log p_{n}}+\frac{3 p_{n}^{2}}{4 \log ^{2} p_{n}}+\frac{7 p_{n}^{2}}{4 \log ^{3} p_{n}}+L(n),
$$

where

$$
L(n)=\frac{44.4 p_{n}^{2}}{8 \log ^{4} p_{n}}+\frac{92.1 p_{n}^{2}}{4 \log ^{5} p_{n}}+\frac{937.5 p_{n}^{2}}{8 \log ^{6} p_{n}}+\frac{5674.5 p_{n}^{2}}{8 \log ^{7} p_{n}}+\frac{79789.5 p_{n}^{2}}{16 \log ^{8} p_{n}} .
$$

Theorem 1.2. For every positive integer $n$, we have

$$
C_{n} \leq \frac{p_{n}^{2}}{2 \log p_{n}}+\frac{3 p_{n}^{2}}{4 \log ^{2} p_{n}}+\frac{7 p_{n}^{2}}{4 \log ^{3} p_{n}}+U(n),
$$

where

$$
U(n)=\frac{45.6 p_{n}^{2}}{8 \log ^{4} p_{n}}+\frac{93.9 p_{n}^{2}}{4 \log ^{5} p_{n}}+\frac{952.5 p_{n}^{2}}{8 \log ^{6} p_{n}}+\frac{5755.5 p_{n}^{2}}{8 \log ^{7} p_{n}}+\frac{116371 p_{n}^{2}}{16 \log ^{8} p_{n}}
$$

\section{Preliminaries}

Let $\pi(x)$ denote the number of primes not exceeding $x$. In 1793, Gauss [3] stated a conjecture concerning the asymptotic behaviour of $\pi(x)$, namely

$$
\pi(x) \sim \operatorname{li}(x) \quad(x \rightarrow \infty)
$$


where the logarithmic integral $\operatorname{li}(x)$ is defined for every real $x \geq 0$ as

$$
\operatorname{li}(x)=\int_{0}^{x} \frac{\mathrm{d} t}{\log t}=\lim _{\varepsilon \rightarrow 0}\left\{\int_{0}^{1-\varepsilon} \frac{\mathrm{d} t}{\log t}+\int_{1+\varepsilon}^{x} \frac{\mathrm{d} t}{\log t}\right\}=\int_{2}^{x} \frac{d t}{\log t}+1.04516 \ldots
$$

Let $m$ be a positive integer. Using integration by parts, (2.2) implies that

$$
\operatorname{li}(x)=\frac{x}{\log x}+\frac{x}{\log ^{2} x}+\frac{2 x}{\log ^{3} x}+\frac{6 x}{\log ^{4} x}+\ldots+\frac{(m-1) ! x}{\log ^{m} x}+O\left(\frac{x}{\log ^{m+1} x}\right) .
$$

The asymptotic formula (2.1) was proved independently by Hadamard [4] and de la ValléePoussin [5], and is known as the Prime Number Theorem. In a later paper, where the existence of a zero-free region for the Riemann zeta-function $\zeta(s)$ to the left of the line $\operatorname{Re}(s)=1$ was proved, de la Vallée-Poussin [6] also estimated the error term in the Prime Number Theorem by showing

$$
\pi(x)=\operatorname{li}(x)+O\left(x e^{-a \sqrt{\log x}}\right),
$$

where $a$ is a positive absolute constant. Applying (2.3) to (2.4), we get

$$
\pi(x)=\frac{x}{\log x}+\frac{x}{\log ^{2} x}+\frac{2 x}{\log ^{3} x}+\frac{6 x}{\log ^{4} x}+\ldots+\frac{(m-1) ! x}{\log ^{m} x}+O\left(\frac{x}{\log ^{m+1} x}\right) .
$$

\section{A proof of Theorem 1.1}

In the following proof of Theorem 1.1, we use a new lower bound for $\pi(x)$.

Proof of Theorem 1.1. First, let $m$ be an integer with $m \geq 2$, and let $a_{2}, \ldots, a_{m}$, and $x_{0}$ be real numbers so that

$$
\pi(x) \geq \frac{x}{\log x}+\sum_{k=2}^{m} \frac{a_{k} x}{\log ^{k} x}
$$

for every $x \geq x_{0}$, and let $y_{0}$ be a real number such that

$$
\operatorname{li}(x) \geq \sum_{j=1}^{m-1} \frac{(j-1) ! x}{\log ^{j} x}
$$

for every $x \geq y_{0}$. The asymptotic formulae (2.5) and (2.3) guarantee the existence of such parameters. In [1, Theorem 13], it is proved that

$$
C_{n} \geq d_{0}+\sum_{k=1}^{m-1}\left(\frac{(k-1) !}{2^{k}}\left(1+2 t_{k-1,1}\right)\right) \frac{p_{n}^{2}}{\log ^{k} p_{n}}
$$

for every integer $n \geq \max \left\{\pi\left(x_{0}\right)+1, \pi\left(\sqrt{y_{0}}\right)+1\right\}$, where $t_{i, j}$ is defined by

$$
t_{i, j}=(j-1) ! \sum_{l=j}^{i} \frac{2^{l-j} a_{l+1}}{l !}
$$

and $d_{0}$ is given by

$$
d_{0}=d_{0}\left(m, a_{2}, \ldots, a_{m}, x_{0}\right)=\int_{2}^{x_{0}} \pi(x) d x-\left(1+2 t_{m-1,1}\right) \operatorname{li}\left(x_{0}^{2}\right)+\sum_{k=1}^{m-1} t_{m-1, k} \frac{x_{0}^{2}}{\log ^{k} x_{0}} .
$$


Now we choose $m=9, a_{2}=1, a_{3}=2, a_{4}=5.85, a_{5}=23.85, a_{6}=119.25, a_{7}=715.5$, $a_{8}=5008.5, a_{9}=0, x_{0}=19027490297$, and $y_{0}=4171$. By [2, Proposition 5], we see that the inequality (3.1) holds for every $x \geq x_{0}$. By [1, Lemma 15], the inequality (3.2) holds for every $x \geq y_{0}$. Substituting these values into (3.3), we get

$$
C_{n} \geq d_{0}+\frac{p_{n}^{2}}{2 \log p_{n}}+\frac{3 p_{n}^{2}}{4 \log ^{2} p_{n}}+\frac{7 p_{n}^{2}}{4 \log ^{3} p_{n}}+L(n)
$$

for every $n \geq 841160648$, where $d_{0}=d_{0}\left(9,1,2,5.85,23.85,119.25,715.5,5008.5,0, x_{0}\right)$ is given by

$$
\begin{aligned}
d_{0}=\int_{2}^{x_{0}} \pi(x) d x-253.3 \operatorname{li}\left(x_{0}^{2}\right)+\frac{126.15 x_{0}^{2}}{\log x_{0}}+\frac{62.575 x_{0}^{2}}{\log ^{2} x_{0}}+\frac{61.575 x_{0}^{2}}{\log ^{3} x_{0}} \\
+\frac{89.4375 x_{0}^{2}}{\log ^{4} x_{0}}+\frac{165.95 x_{0}^{2}}{\log ^{5} x_{0}}+\frac{357.75 x_{0}^{2}}{\log ^{6} x_{0}}+\frac{715.5 x_{0}^{2}}{\log ^{7} x_{0}} .
\end{aligned}
$$

Hence it suffices to show that $d_{0}>0$. By [1, Lemma 16], we have

$$
\operatorname{li}(x) \leq \frac{x}{\log x}+\frac{x}{\log ^{2} x}+\frac{2 x}{\log ^{3} x}+\frac{6 x}{\log ^{4} x}+\frac{24 x}{\log ^{5} x}+\frac{120 x}{\log ^{6} x}+\frac{900 x}{\log ^{7} x}
$$

for every $x \geq 10^{16}$. Applying (3.6) to (3.5), we get

$$
\begin{aligned}
d_{0} \geq \int_{2}^{x_{0}} \pi(x) d x-\frac{x_{0}^{2}}{2 \log x_{0}}-\frac{3 x_{0}^{2}}{4 \log ^{2} x_{0}}-\frac{7 x_{0}^{2}}{4 \log ^{3} x_{0}}-\frac{5.55 x_{0}^{2}}{\log ^{4} x_{0}}-\frac{23.025 x_{0}^{2}}{\log ^{5} x_{0}} \\
\quad-\frac{117.1875 x_{0}^{2}}{\log ^{6} x_{0}}-\frac{1065.515625 x_{0}^{2}}{\log ^{7} x_{0}} .
\end{aligned}
$$

We can show by a straightforward calculation that $d_{0} \geq 1.12 \cdot 10^{13}>0$. For every integer $n$ satisfying $440200309 \leq n \leq 841160647$, we check the inequality with a computer.

\section{A proof of Theorem 1.2}

Next, we use a recent result concerning an upper bound for the prime counting function $\pi(x)$ to establish the required inequality stated in Theorem 1.2.

Proof of Theorem 1.2. Let $m$ be an integer with $m \geq 2$, let $a_{2}, \ldots, a_{m}$, and $x_{1}$ be real numbers so that

$$
\pi(x) \leq \frac{x}{\log x}+\sum_{k=2}^{m} \frac{a_{k} x}{\log ^{k} x}
$$

for every $x \geq x_{1}$ and let $\lambda$ and $y_{1}$ be real numbers so that

$$
\operatorname{li}(x) \leq \sum_{j=1}^{m-2} \frac{(j-1) ! x}{\log ^{j} x}+\frac{\lambda x}{\log ^{m-1} x}
$$


for every $x \geq y_{1}$. Again, the asymptotic formulae (2.5) and (2.3) guarantee the existence of such parameters. In [1, Theorem 14] it is proved that the inequality

$$
\begin{aligned}
C_{n} \leq d_{1} & +\sum_{k=1}^{m-2}\left(\frac{(k-1) !}{2^{k}}\left(1+2 t_{k-1,1}\right)\right) \frac{p_{n}^{2}}{\log ^{k} p_{n}} \\
& +\left(\frac{\left(1+2 t_{m-1,1}\right) \lambda}{2^{m-1}}-\frac{a_{m}}{m-1}\right) \frac{p_{n}^{2}}{\log ^{m-1} p_{n}}
\end{aligned}
$$

holds for every integer $n \geq \max \left\{\pi\left(x_{1}\right)+1, \pi\left(\sqrt{y_{1}}\right)+1\right\}$, where $t_{i, j}$ is defined by (3.4) and

$$
d_{1}=d_{1}\left(m, a_{2}, \ldots, a_{m}, x_{1}\right)=\int_{2}^{x_{1}} \pi(x) d x-\left(1+2 t_{m-1,1}\right) \operatorname{li}\left(x_{1}^{2}\right)+\sum_{k=1}^{m-1} t_{m-1, k} \frac{x_{1}^{2}}{\log ^{k} x_{1}} .
$$

Next we choose $m=9, a_{2}=1, a_{3}=2, a_{4}=6.15, a_{5}=24.15, a_{6}=120.75, a_{7}=724.5$, $a_{8}=6601, a_{9}=0, \lambda=6300, x_{1}=13$, and $y_{1}=10^{18}$. By [2, Proposition 3], we get that the inequality (4.1) holds for every $x \geq x_{1}$ and by [1, Lemma 19], we see that (4.2) holds for every $y \geq y_{1}$. By substituting these values into (4.3), we get

$$
C_{n} \leq d_{1}+\frac{p_{n}^{2}}{2 \log p_{n}}+\frac{3 p_{n}^{2}}{4 \log ^{2} p_{n}}+\frac{7 p_{n}^{2}}{4 \log ^{3} p_{n}}+U(n)-\frac{0.375 p_{n}^{2}}{16 \log ^{8} p_{n}}
$$

for every integer $n \geq 50847535$, where $d_{1}=d_{1}\left(9,1,2,6.15,24.15,120.75,724.5,6601,0, x_{1}\right)$ is given by

$$
\begin{aligned}
d_{1}=\int_{2}^{x_{1}} \pi(x) d x-\frac{26599}{90} \operatorname{li}\left(x_{1}^{2}\right)+\frac{26509 x_{1}^{2}}{180 \log x_{1}}+\frac{26329 x_{1}^{2}}{360 \log ^{2} x_{1}}+\frac{25969 x_{1}^{2}}{360 \log ^{3} x_{1}} \\
+\frac{25231 x_{1}^{2}}{240 \log ^{4} x_{1}}+\frac{11891 x_{1}^{2}}{60 \log ^{5} x_{1}}+\frac{5221 x_{1}^{2}}{12 \log ^{6} x_{1}}+\frac{943 x_{1}^{2}}{\log ^{7} x_{1}}
\end{aligned}
$$

A direct computation gives $d_{1} \leq 453$. We define $f(x)=0.375 x^{2} /\left(16 \log ^{8} x\right)-453$. Since $f^{\prime}(x) \geq 0$ for every $x \geq e^{4}$ and $f(9187322)>0$, we get $f\left(p_{n}\right) \geq 0$ for every integer $n \geq$ $\pi(9187322)+1=614124$. Now we can use (4.4) to obtain the desired inequality for every integer $n \geq 50847535$. Finally, we check the remaining cases with a computer.

\section{Acknowledgements}

I would like to thank the anonymous reviewers for their careful reading of this paper.

\section{References}

[1] Axler, C. (2015). On a sequence involving prime numbers, J. Integer Seq., 18, no. 7, Article 15.7.6, $13 \mathrm{pp}$.

[2] Axler, C. (2018). New estimates for some functions defined over primes, Integers, 18, Paper No. A52, 21 pp. 
[3] Gauß, C. F. (1876). Werke, 2 ed., Königlichen Gesellschaft der Wissenschaften, Göttingen.

[4] Hadamard, J. (1896). Sur la distribution des zéros de la fonction $\zeta(s)$ et ses conséquences arithmétiques, Bull. Soc. Math. France, 24, 199-220.

[5] de la Vallée Poussin, C.-J. (1896). Recherches analytiques la théorie des nombres premiers, Ann. Soc. scient. Bruxelles, 20, 183-256.

[6] de la Vallée Poussin, C.-J. (1899). Sur la fonction $\zeta(s)$ de Riemann et le nombre des nombres premiers inférieurs à une limite donnée, Mem. Couronnés de l'Acad. Roy. Sci. Bruxelles, 59, $1-74$. 